\title{
Modalidad de educación mixta. Un modelo interdisciplinario orientado al aprendizaje consciente y autorregulado del estudiante de postgrado
}

\author{
Mixed education mode. An interdisciplinary model oriented to the \\ conscious and self-regulated learning of the postgraduate student
}

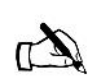

Ana Acevedo

profaanaacevedo@gmail.com

Código ORCID: oooo-ooo1-5144-9519

Universidad Pedagógica Experimental Libertador, Venezuela
Artículo recibido en febrero 2019 Arbitrado en marzo 2019 Publicado en mayo 2019

\section{Resumen}

El objetivo de la investigación fue describir el proceso para generar un modelo interdisciplinario para la modalidad mixta, orientado al aprendizaje consciente y autorregulado del estudiante de postgrado, con base en las concepciones de estudiantes y docentes. La metodología estuvo enmarcada en el paradigma cualitativo, desde una perspectiva fenomenológica y un diseño de estudio de caso. Para el análisis de los resultados se seleccionó la teoría fundamentada y el método comparativo constante. Se aplicó la entrevista en profundidad a estudiantes y docentes del Instituto Pedagógico de Miranda de la Universidad Pedagógica Experimental Libertador. La técnica utilizada fue la entrevista en profundidad y el instrumento, un guión de entrevista. El hallazgo más importante fue que la modalidad de educación mixta representa la búsqueda de soluciones a las necesidades de formación, integrando los elementos de la modalidad presencial y virtual. Todo esto se logra con docentes competentes tecnológica y pedagógicamente.

\footnotetext{
Abstract

The objective of the research was to describe the process to generate an interdisciplinary model for the mixed modality, oriented to the conscious and self-regulated learning of the postgraduate student, based on the conceptions of students and teachers. The methodology was framed in the qualitative paradigm, from a phenomenological perspective and a case study design. For the analysis of the results, the grounded theory and the constant comparative method were selected. The in-depth interview was applied to students and teachers of the Miranda Pedagogical Institute of the Libertador Experimental Pedagogical University. The technique used was the in-depth interview and the instrument, an interview script. The most important finding was that the mixed education modality represents the search for solutions to the training needs, integrating the elements of the classroom and virtual modality. All this is achieved with competent teachers technologically and pedagogically.
}

Palabras clave:

Modelo pedagógico, modalidad de educación mixta, postgrado

\section{Keywords:}

Pedagogical model, mixed education modality, postgraduate 
INTRODUCCIÓN

En la actualidad uno de los grandes retos a los que debe enfrentarse la educación universitaria es saber responder de manera adecuada a la necesidad de formación a lo largo de toda la vida. E1 desafío de la universidad en estos tiempos implica abandonar la idea de entidad transmisora de conocimiento científico elaborado para dar paso a la mediación, brindar las ayudas oportunas para que el estudiante "aprenda a aprender" lo que, sin lugar a dudas, implicará un cambio radical en el modelo educativo universitario actual. Por tanto, el docente deja de ser el centro de atención, pasando a ser el estudiante el verdadero protagonista de los procesos de aprendizaje.

Partiendo de las ideas expresadas, es importante mencionar que cada día cobra mayor vigencia la educación a distancia como alternativa válida para llevar a cabo los procesos de enseñanza y aprendizaje en el sector universitario. Por lo tanto, el Estado venezolano en el año 2008 aprobó el Programa de Formación Docente en Educación a Distancia con el "fin de contribuir de manera estratégica, articulada y sistémica, con el desarrollo, implementación y consolidación de esta modalidad de estudio en las Instituciones de Educación Universitaria (IEU)" (OPSU, 2008, p. 1).

En el mencionado programa se destaca la necesidad de atender al profesorado en cuanto a su formación para poder afrontar los nuevos entornos de trabajo y así asumir con calidad y pertinencia su rol en la modalidad de educación a distancia. Se debe proporcionar las competencias teóricas, pedagógicas, didácticas y tecnológicas acordes con los enfoques, estrategias instruccionales y de evaluación que se adecuen a esta modalidad educativa.

Por lo tanto, la educación universitaria debe ajustarse a los cambios que trae consigo la incorporación de las TIC, hacer grandes esfuerzos que permitan la utilización de las tecnologías en su oferta educativa y en sus métodos docentes. Al respecto Sangrá (2002) destaca:

Con el objetivo de hacer llegar la
educación a todo aquel que la
necesita, aparecieron las prácticas
de educación a distancia. Estas
prácticas han exigido siempre la
existencia de un elemento
mediador entre el docente y el
discente. Si históricamente nos
referimos al correo convencional,
que establecía una relación
epistolar entre el profesor y el
estudiante, con el tiempo hemos
ido introduciendo nuevas
tecnologías que, por su coste y su
accesibilidad, nos permiten
evolucionar en esta relación a
distancia. (p. 2)

De acuerdo con las ideas expresadas por el autor, la educación a distancia con el apoyo de las tecnologías reduce los obstáculos de espacio y tiempo, se recurre a métodos, técnicas y recursos que elevan la productividad y la flexibilidad en los procesos de enseñanza y aprendizaje. Las TIC pueden desempeñar, fundamentalmente, un papel de apoyo a la docencia, mayor interacción entre profesores y estudiantes, formación a lo largo de la vida, ya sea por las necesidades de actualización, mayor 
especialización o reorientación de la carrera profesional.

Por otro lado, es importante destacar que con el auge de Internet se produjo un incremento generalizado del uso de las TIC en las instituciones educativas y, particularmente, en las universidades, estas han mostrado un progresivo interés en la incorporación de las tecnologías y en la actividad docente, debido a varias razones expresadas por Sigalés (2004): a) la accesibilidad que proporcionan las TIC; b) su potencial pedagógico; c) la facilidad de manejo por parte de profesores y estudiantes; d) la creciente presión social para la incorporación de dichas tecnologías; e) la necesidad de superar las limitaciones espacio-temporales de la docencia presencial, y f) las nuevas oportunidades que proporciona un espacio universitario global.

La incorporación de las TIC en el ámbito de la formación universitaria constituye una de las inquietudes comunes de estas instituciones de educación que aspiran a modernizar sus estructuras organizativas y propuestas de formación, nivel en que se desarrolla la presente investigación.

En la educación a distancia existen diversos modelos de formación con apoyo de las TIC, entre ellos tenemos el modelo de educación mixta, también llamado semipresencial, b-learning o blendedlearning, aprendizaje mezclado, aprendizaje híbrido, aprendizaje flexible, existen muchas denominaciones pero la idea primordial de estos modelos subyace en la selección de las vías y medios más adecuados para cada necesidad educativa.

De allí que el propósito de esta investigación se centró en describir cómo fue el proceso que permitió generar un modelo interdisciplinario para la modalidad de educación mixta, orientado al aprendizaje consciente y autorregulado del estudiante de postgrado del Instituto Pedagógico de Miranda José Manuel Siso Martínez (IPMJMSM), perteneciente a la Universidad Pedagógica Experimental Libertador (UPEL), con base en las concepciones de los estudiantes y docentes, a fin de fortalecer y mejorar los estudios de postgrado que en el caso de los estudiantes les permitirá ser autónomos y capaces de autorregular sus estudios y favorecer la reflexión metacognitiva. Para los docentes este modelo les aportará herramientas pedagógicas que ayudarán a facilitar de manera combinada los procesos de enseñanza y aprendizaje.

Para cumplir con el objetivo general se formularon los siguientes objetivos específicos:

1. Interpretar las concepciones que poseen los estudiantes y los docentes en relación con un modelo pedagógico de educación mixta para el nivel de postgrado en el Instituto Pedagógico de Miranda José Manuel Siso Martínez.

2. Establecer los elementos teóricos y empíricos que configuran un modelo pedagógico de educación mixta para el nivel de postgrado en el Instituto Pedagógico de Miranda José Manuel Siso Martínez.

3. Diseñar el modelo pedagógico de educación mixta para el nivel de postgrado en el Instituto Pedagógico de Miranda José Manuel Siso Martínez 
MÉTODO

El tipo de investigación que se asumió en esta construcción teórica se encuentra enmarcado dentro del enfoque interpretativo. Según Cook y Reichardt (1997) este permite un acercamiento más global y comprensivo de la realidad social y a medida que se avanza en la investigación se determinan nuevos fenómenos para el análisis. Se promueve una forma particular y lógica de estudiar los fenómenos sociales a través de la realidad estudiada.

En cuanto al diseño de la investigación, se desarrolló bajo una investigación de campo fundamentada en un estudio de casos, de carácter descriptivo e interpretativo, porque la información fue recogida en la realidad donde se produjo; más concretamente, en los escenarios del Instituto Pedagógico de Miranda José Manuel Siso Martínez (IPMJMSM) correspondiente a la Universidad Pedagógica Experimental Libertador (UPEL); específicamente con estudiantes del postgrado, nuevo ingreso, avanzados, y egresados; así como profesores ordinarios y contratados, de este mismo nivel, sin descontextualizar los datos aislándolos de su contexto natural.

Los criterios para la selección de los informantes según Strauss y Corbin (2002) se componen en mecanismos de comprensión adecuados y no representativos en términos estadísticos, lo cual implica asumir la realidad como compleja donde la diversidad de perspectivas en una situación son legítimas.

En este estudio los criterios iniciales que permitieron determinar a los sujetos en un primer lapso fueron: a) tener experiencia como estudiante o profesor en algún subprograma administrado por la
Subdirección de Investigación y Postgrado en los últimos tres años; y b) poseer la disposición (emotiva y de tiempo) de ser abordado por la investigadora para su entrevista. Estos criterios iniciales permitieron abordar la realidad objeto a estudio y seleccionar inicialmente diez (10) profesores, que se clasificaron en: a) cuatro (4) profesores ordinarios, es decir que forma parte de la planta profesoral de la UPEL, y fueron denominados como POU; b) seis (6) profesores contratados que prestan sus servicios por honorarios profesionales. Son profesores contratados $\mathrm{y}$ se designaron como PC, en total se entrevistaron inicialmente diez (10) profesores que administran cursos en los diferentes Subprogramas del postgrado. En cuanto a los estudiantes, se entrevistaron originariamente a quince (15) sujetos de la siguiente manera: a) ocho (8) estudiantes regulares inscritos que estaban iniciando sus estudios en el postgrado, fueron denominados con las siglas ERIE; b) cinco (5) estudiantes regulares avanzados que en su mayoría terminaron la escolaridad y estaban en la elaboración del trabajo de grado con las siglas ERA y, por último; c) dos (2) estudiantes egresados de los estudios de postgrado, los cuales fueron denominados con las siglas EE.

La técnica utilizada fue la entrevista en profundidad que requiere de preguntas abiertas, escuchar y registrar las respuestas y luego complementar con preguntas relevantes. Esta se realizó de forma individual a diez (10) profesores que administran cursos en el nivel de postgrado y a quince (15) estudiantes que han hecho y hacen vida en nivel de postgrado. Pero como ya se ha dicho anteriormente, por el fenómeno de 
saturación teórica fueron considerados diez (10) informante como claves (cinco profesores y cinco estudiantes).

El instrumento que se empleó con esta técnica fue el guión de entrevista, el cual según Taylor y Bogdan (1990) se trata de una lista de áreas generales que deben cubrirse con cada informante y que deben derivarse de los propósitos u objetivos de la investigación y no pueden responder a formulaciones textuales de preguntas ni sugerir, mucho menos, las opciones de respuestas. Las preguntas se redactaron partiendo de la primera interrogante de la investigación, transcritas de manera general, aún cuando estas preguntas se presentan en un orden específico en el momento de transcribir la entrevista, durante la realización de esta se flexibilizó el orden con la finalidad de hacer más espontáneas las respuestas. El formato o guía utilizado para realizar el guion de entrevista fue una adaptación que se hizo de Feo (2013).

El desarrollo de la investigación atendió a tres momentos:

Primer momento. Interpretación de las concepciones de docentes y estudiantes. Se realizó la revisión y análisis de la literatura relacionada con el objeto de estudio, tales como: textos, trabajos de investigación y revistas especializadas, así como también fuentes audiovisuales y electrónicas. Se hicieron las lecturas exploratorias a fin de determinar y considerar su validez con el contenido.

Segundo momento. Elementos teóricos y empíricos del modelo. $\mathrm{Al}$ analizar e interpretar la teoría emergente derivada de la aplicación del método comparativo constante se determinó que para diseñar el modelo pedagógico que diera respuestas favorables a la realidad existente entre los estudiantes y los profesores que hacen vida en el postgrado del IPMJMSM el modelo pedagógico debía constituirse con base en los elementos teóricos y empíricos que dan soporte para el diseño del modelo pedagógico.

Tercer momento. Diseño del modelo pedagógico. En la construcción de este momento se emplearon los supuestos teóricos y empíricos emergentes en la teoría vigente referencial asumida y de los hallazgos resultantes de la aplicación del método comparativo constante a la información recabada de los diez (10) informantes claves.

\section{RESULTADOS}

El análisis cualitativo de los datos se realizó enfatizando en la inducción analítica a partir de la teoría fundamentada y el empleo del método comparativo constante a objeto de clarificar los procesos de descripción, codificación e interpretación, llevados a cabo con el propósito de descubrir conceptos y relaciones para luego organizarlos en esquemas teóricos explicativos.

Primer momento de la investigación. Interpretación de las concepciones de estudiantes y docentes

\section{Categoría 1.E. Concepción acerca de la modalidad de educación mixta de los estudiantes}

Definición: creencias, opiniones, ideas $\mathrm{y}$ reflexiones que tienen los 
estudiantes del nivel de postgrado acerca de la educación mixta.

\section{Subcategorías:}

La subcategoría 1.1.E.- Concepción teórica de la modalidad mixta de educación mixta hace referencia a los conocimientos teóricos que manejan los estudiantes del nivel de postgrado producto de la información que tienen sobre los avances e incorporación de las tecnologías en el ámbito educativo, perciben que la modalidad de educación mixta es aquella que está conformada por "ambientes de aprendizaje combinados o mixtos" o "educación semipresencial" donde lo importante es combinar los elementos que nos ofrece la educación virtual con la educación presencial. Destacan que es importante tomar en cuenta los estilos de aprendizaje en una modalidad mixta para no limitar al estudiante en lo presencial o en lo virtual.

En lo relativo a la subcategoría 1.2.E.Concepción empírica de la modalidad de educación mixta, la investigadora la define como la experiencia, percepción y opinión que tienen los estudiantes sobre la modalidad. Los estudiantes consideran que la modalidad te permite desempeñarte de diferentes formas, tanto en lo presencial como en lo virtual y destacan que se debe "ser bien estratégico para combinar ambas" donde lo presencial no esté por encima de lo virtual o viceversa sino que debe existir un equilibrio en la combinación de ambas modalidades.

Otro aspecto bien interesante que surge de los hallazgos es la "flexibilidad para el aprendiz" que proporciona la modalidad, pues subrayan el hecho de que los horarios son muchos más flexibles y personalizados que permiten estudiar desde la comodidad de su oficina, de su hogar haciendo uso de la diversidad de recursos tecnológicos que existen en la actualidad.

\section{Categoría 2.E. Implicaciones prácticas de la modalidad educación mixta}

Definición: Se refiere a la trascendencia, utilidad, beneficios $y$ alcances que tendría la aplicación de la modalidad de educación mixta para el nivel de postgrado.

\section{Subcategorías:}

La subcategoría 2.1.E. -Beneficios de la modalidad de educación mixta permite reconocer las ventajas que ofrece la aplicación de la modalidad de educación mixta para los estudios de postgrado ya que los estudiantes no tendrían la necesidad de asistir de manera presencial a clase. Ellos manifiestan que la modalidad mixta propicia la "reducción de costos $y$ tiempo" ya que el estudiante puede gerenciar su tiempo de acuerdo a sus actividades personales y laborales y así muchos no abandonarían el postgrado por falta de tiempo.

La subcategoría 2.2. E.- Desventaja de la modalidad de educación mixta surge como la circunstancia que impide en la actualidad la implementación de la modalidad de educación en los estudios de postgrado del IPMJMSM, los estudiantes expresan y perciben que: "Falta de formación en TIC de profesores", "Falta de disposición para el uso de las TIC" y "Falta de recursos tecnológicos" como elementos que 
deben estar presentes en la modalidad de educación mixta. La falta de formación en TIC de los profesores es un elemento bien importante ya que "si el docente no está bien preparado no se obtendrían buenos resultados" y ocurre que cada profesor utiliza las TIC de acuerdo a lo que conoce porque no existe un plan de formación para los profesores que administran cursos en el nivel de postgrado.

La subcategoría 2.3.E-Elementos de la comunicación virtual corresponde a los medios de comunicación interpersonal a través de la modalidad virtual, que adoptan dos formas: sincrónica $\mathrm{y}$ asincrónica. La primera en la que los usuarios a través de una red telemática coinciden en el tiempo y se comunican entre sí, y la segunda donde los participantes utilizan el sistema de comunicación en tiempos diferentes.

Los estudiantes aprecian mucho la utilización de la comunicación sincrónica a través de chat, vía Skype, videoconferencias donde todos los involucrados puedan conectarse al mismo tiempo y con un propósito. En la comunicación asincrónica destacan el uso del foro Web, la creación de blog y páginas web como herramientas de aprendizaje y sobre todo porque lo pueden hacer en cualquier momento. Es importante destacar el testimonio de un estudiante que propone trabajar en el aspecto virtual "con entornos educativos $3 D$ a través de avatares donde puedas dar contenidos, subir material, entre otros", aquí está presente la formación que ha recibido lo que le permite expresarse con propiedad de elementos indispensables en comunicación virtual.
La subcategoría 2.4.E. -Componentes de un modelo de educación mixta constituye los elementos que de acuerdo con la opinión de los informantes deben considerarse al momento de diseñar un modelo que esté fundamentado en la modalidad mixta o combinada. Es oportuno recalcar que casi en su totalidad los estudiantes coincidieron en que los componentes de un modelo mixto son una buena "Plataforma", "Formación en TIC en el ámbito educativo" y "Cursos y talleres".

La subcategoría 2.5.E.- Rol del estudiante en la modalidad de educación mixta es considerado como el papel que desempeña un discente en un modelo de aprendizaje combinado. Se destacan dos indicadores "estudiante activo" y "estudiante autónomo" para la autora los hallazgos están focalizados en que el estudiante es responsable de su aprendizaje, más proactivo, buscar las herramientas para aprender los aspectos tecnológicos, no depender tanto del profesor, un estudiante autónomo; es evidente que el estudiante de postgrado tiene muy definida cuál es su actuación en este tipo de modalidad.

Los estudiantes de postgrado son profesionales con experiencias y aprendizaje previos que tiene que "desaprender" para aprender nuevas estrategias de aprendizaje que le permitan desempeñarse con autonomía en una modalidad mixta, tendrán que convertirse en aprendices estratégicos, capaces de autorregular su propio aprendizaje y el manejo de las nuevas tecnologías de la información y comunicación, todo ello con responsabilidad y autonomía (Monereo, 2003). 
Categoría 3.E.-Tecnologías de la Información y Comunicación en el nivel de postgrado

Definición: Se refiere al uso, formación y aplicación de medios y recursos que ofrecen las TIC en el nivel de postgrado.

\section{Subcategorías:}

La subcategoría 3.1.E.- Aplicación de TIC en el Postgrado nos proporciona información acerca de los "recursos tecnológicos que usan los estudiantes en el postgrado" aquí se evidencia que los recursos que más utilizan para realizar estudios de postgrado son: Internet a través del correo electrónico para enviar y recibir información, redes sociales como Twitter y Facebook, buscadores como Google, programas Word, PowerPoint entre otros. Sin embargo, el correo electrónico es el que tiene más uso como medio de comunicación entre profesores y estudiantes.

Siguiendo con la subcategoría 3.2.E.Tipo de formación en TIC que poseen los estudiantes de postgrado, tenemos que tiene una "formación empirica" que han desarrollado por ensayo y error, por iniciativa propia, de allí la importancia que el instituto le ofrezca cursos introductorios o talleres de formación para adquirir competencias en el uso de las TIC. Son pocos los que han recibido "formación académica". El informante que recibió formación en Fatla (Fundación para la Actualización Tecnológica de Latinoamérica) es profesor ordinario del instituto y es estudiante de postgrado $\mathrm{y}$ otro de los informantes recibió en pregrado un curso optativo sobre Informática Aplicada a la Educación.

\section{Categoría 1.D. Concepción acerca de} la modalidad de educación mixta de los docentes

Definición: Creencias, opiniones, ideas y reflexiones que tienen los profesores del nivel de postgrado acerca de la educación mixta.

\section{Subcategorías:}

La subcategoría 1.1.D.-Concepciones teóricas de la modalidad de educación mixta los docentes en sus opiniones, ideas y reflexiones la definen de acuerdo a lo que plantea la diversa literatura sobre el tema, de allí surgen dos indicadores: "Combinación de lo presencial y lo virtual" y "Enseñanza presencial con apoyo en medios electrónicos" que expresan claramente a que se refiere dicha modalidad. En cuanto al primer indicador exponen que la modalidad mixta "incluye un elemento nuevo que es la virtualidad" y "maneja la presencialidad y la virtualidad", la modalidad de educación mixta como se plantea en esta investigación es la combinación de las actividades presenciales con actividades virtuales donde se busca lo mejor de los aspectos presenciales como virtuales en beneficio de un proceso de enseñanza y aprendizaje óptimos, por lo tanto la conceptualización que manejan los docentes es cónsona con los principios que maneja la modalidad.

La segunda subcategoría 1.2.D.Concepciones empiricas de la modalidad de educación mixta contiene las experiencias que han tenido los profesores en su 
desarrollo y actuación profesional en la mencionada modalidad. Se originan los indicadores "Asesoría con complemento virtual" y "Experiencia en la modalidad mixta". En el primer indicador es un modelo donde se ofrecen tutorías presenciales además de las propias de los sistemas virtuales o digitales. En el segundo indicador se evidencia que conocen la modalidad porque han administrado cursos en pregrado llamados a distancia y se apoyan con los recursos que les ofrece la virtualidad; también han realizados sus estudios en el desarrollo de su carrera profesional en la modalidad mixta.

\section{Categoría 2.D. -Implicaciones prácticas de la modalidad de educación mixta}

Definición: Se refiere a la trascendencia, utilidad, beneficios $\mathrm{y}$ alcances que tendría la aplicación de la modalidad de educación mixta para el nivel de postgrado

\section{Subcategorías:}

En la subcategoría 2.1.D. -Beneficios de la modalidad de educación mixta surgieron tres indicadores que son los siguientes: "Reducción de costos, tiempo y utilización eficiente de los espacios", "Aprendizaje Significativo y Aprendizaje Estratégico" y "Flexibilidad en la modalidad". En cuanto al primer indicador la modalidad tiene como ventaja el ahorro económico de traslado y de recursos materiales que se requieren para realizar estudios de postgrado, también el ahorro de tiempo que ofrece la virtualidad porque se puede estudiar y realizar las actividades individuales $\mathrm{y}$ grupales desde cualquier lugar, es decir el "ahorro de distancia" como lo llaman algunos profesores. Por otro lado, la utilización eficiente de los espacios físico y así se podría atender mayor cantidad de estudiantes que desean ingresar al postgrado.

Es oportuno resaltar que los estudiantes de postgrado del IPMJMSM tienen la limitante de las grandes distancias que tienen que transitar para poder llegar al lugar de estudio y la necesidad de invertir mucho tiempo y recursos económicos para cumplir con los horarios y actividades propias de la modalidad presencial. Mortera (2007) sostiene que la instrucción cara a cara o presencial a menudo es mucho más costosa que la educación a distancia (formato mixto o semipresencial o elearning).

Siguiendo con la subcategoría 2.2.D. Desventaja de la modalidad educación mixta tenemos de acuerdo con los testimonios de los docentes los siguientes indicadores que se mencionan a continuación: "Falta de formación docente en TIC" y "La no aplicación de políticas institucionales"; sin embargo los informantes manifestaban que la modalidad de educación mixta tiene más ventajas que desventajas. Con respecto al primer indicador es evidente que la falta o ausencia de competencias docentes en el manejo y aplicación de las tecnologías producen inconvenientes al momento de proponer y ejecutar proyectos en la modalidad mixta.

Un elemento clave para promover la innovación apoyada en TIC dentro de las instituciones educativas, se les reconoce como actores fundamentales del sistema educativo, y para los que se debe 
garantizar, entre otras cosas, una formación y capacitación permanente en provecho de las mejoras de la educación. Salinas (2004) enfatiza que el rol del docente al igual que el de los estudiantes cambia en un ambiente enriquecido por las TIC. Sus conocimientos y destrezas son esenciales para el buen funcionamiento de cualquier programa y en especial en formación en recursos tecnológicos aplicados a la educación.

La UPEL (2011) en el Documento Base de Currículo señala en su concepción de currículo como espacio público pretende dar respuestas diferentes demandas y entre ellas se encuentra la "Incorporación de las TIC en todos los proceso" con responsabilidad social que permita mejorar continuamente la formación integral y el desarrollo de competencias ciudadanas.

E1 Vicerrectorado de Investigación y Postgrado diseño el "Proyecto para implementar la "Educación Universitaria a Distancia a través de los Subprogramas de Postgrados en la UPEL" con el propósito de construir los diseños curriculares atendiendo a los perfiles y competencias requeridas e incorporando la modalidad a distancia en la administración curricular, la virtualización de los cursos y la evaluación en correspondencia con las competencias (UPEL, 2010).

Siguiendo el análisis e interpretación tenemos la subcategoría 2.3.D-Elementos de la comunicación virtual estos pueden definirse como los recursos que se utilizan en las actividades virtuales en una modalidad mixta, sin embargo la autora de la presente investigación los clasifica en sus respectivos indicadores como "sincronos" y "asincronos" porque estos medios en su momento de utilizarlos responden a un tiempo, es decir, posibilita la interactividad síncrona y asíncrona. A través de la presencia de éstos medios tecnológicos aumentan los elementos de diálogo, autonomía, autorregulación y retroalimentación en el proceso de enseñanza y aprendizaje (Lobo, 2009).

En la interactividad síncrona los docentes destacan su gusto por las video conferencias, el Skype, el chat; en la interactividad asíncrona el foro web, páginas web, blog, el envío de información por correo electrónico. Los canales de comunicación y los recursos en lo que se apoyan la Educación a Distancia permite la interacción de espacio y tiempo sin ninguna limitante, es decir, tiene un carácter flexible, de esta manera se fomenta el aprendizaje colaborativo, cooperativo y tutelado entre los estudiantes (UNESCO, 2010).

En lo que respecta a la subcategoría 2.4.D. -Componentes de un modelo de educación mixta se origina de los hallazgos más significativos aportados por los profesores, ellos estiman que en un modelo educativo mixto en el IPMJMSM deben considerarse una buena "Plataforma y equipos", "Cursos de inducción y talleres en TIC" y "Competencias docentes en TIC".

En el ámbito de la sociedad de la información, la formación del profesorado debe diseñarse de tal modo que permita crear las competencias adecuadas para formar profesionales que no sólo dominen la didáctica especifica de las diferentes materiales sino también una serie de elementos educativos transversales que todo profesional de la educación debe adquirir (Gros y Silva, 2005). 
La subcategoría 2.5.D.-Rol del docente en la modalidad mixta considerado como el papel que desempeña el profesor en la modalidad, sin embargo, es relevante señalar que el rol que cumple en la modalidad mixta es diferente al de la modalidad presencial, deja de ser la fuente del conocimiento para convertirse en un facilitador, mediador, orientador y asesor del aprendizaje, así lo muestran las opiniones emitidas por los informantes. Es responsable de guiar, revisar, evaluar, retroalimentar a sus estudiantes en el proceso de aprendizaje.

Es evidente que el docente es un promotor de aprendizajes, guía y reorienta el proceso de aprendizaje motivando y promoviendo el interés de sus alumnos, es decir se convierte en un facilitador del aprendizaje. Por tanto, el rol del docente en la modalidad mixta impone una nueva pedagogía basada en la combinación de la interactividad y la presencialidad en la personalización del proceso de enseñanza y aprendizaje y en el desarrollo de la capacidad de aprender de manera autónoma.

Del Mastro (2005) destaca que el rol principal del profesor tutor al brindar las ayudas pedagógicas necesarias es que sus estudiantes aprendan a aprender, creando múltiples zonas de desarrollo próximo en relación con las maneras de entender y enfrentarse al proceso de estudio y aprendizaje en la modalidad en un contexto virtual.

Categoría 3.D. -Tecnologías de la Información y Comunicación en el nivel de postgrado

Definición: Se refiere al uso, formación y aplicación de medios y recursos que ofrecen las TIC en el nivel de postgrado.

\section{Subcategorías:}

En la subcategoría 3.1.D. -Tipo de Formación en TIC está referida a la formación que han recibido los profesores del IPMJMSM durante su trayectoria profesional docente. De los hallazgos encontrados se desprenden dos indicadores que señalan una "Formación empírica" y una "Formación académica". Pero es relevante mencionar que la mayoría de los informantes indican que su formación ha sido empírica a través del "ensayo y error", por "iniciativa propia", "por apoyo de mis hijos y esposa" "explorando con algunos de mis compañeros" estos son algunos de los testimonios que expresados. En la formación académica lo han realizado por medio de talleres, cursos y porque les agrada la tecnología, pero son muy pocos los informantes que han recibido este tipo de formación.

Por lo antes mencionado se puede interpretar y reflexionar acerca de la formación necesaria que debe tener el docente para el buen desempeño en un contexto signado por las TIC y en especial en una modalidad mixta; el docente debe poseer formación pedagógica aplicada a las tecnologías para ayudar a desarrollar un saber colectivo, dando autonomía al alumno y desarrollando el aprendizaje colaborativo. Según Gisbert (2000) para que el docente desempeñe su rol con eficiencia debe tener una serie de competencias, algunas de las cuales serán nuevas y otras serán una modificación de las que venía desempeñando; estas competencias pueden resumirse en torno a tres dimensiones fundamentales: 
Saber, conocimiento sobre las competencias que ha de asumir; (2) Saber hacer, saber desarrollar los métodos de enseñanza, de tutoría y de monitorización mediante el uso de herramientas tecnológicas y (3) Saber ser, contar con habilidades y destrezas comunicativas y sociales: retroalimentación, trabajo en equipo, negociación y relación interpersonal.

La subcategoría 3.2.D.- Aplicación de TIC en el nivel de postgrado emerge como el uso que le dan los docentes a los recursos y medios que nos ofrecen las nuevas tecnologías en el nivel de postgrado para administrar sus cursos y cómo ellos interpretan se aprovechan estos recursos en dicho nivel. Para esta subcategoría surgen los siguientes indicadores "Recursos tecnológicos que usan los profesores de postgrado" y "Poco aprovechamiento de las TIC en postgrado".

Segundo momento de la investigación. Elementos teóricos y empíricos del MOPEM

Acevedo (2019) presentó en el libro titulado Modelo pedagógico fundamentado en la modalidad de educación mixta para el nivel de postgrado los once (11) elementos teóricos y los once (11) elementos empíricos que corresponden al modelo propuesto, producto del informe de investigación del cual se derivó este artículo. Allí se recomienda la posibilidad de que el instituto pedagógico ofrezca a los interesados cursos introductorios $\mathrm{o}$ talleres de formación, con el fin de que se adquieran las respectivas competencias para el manejo de las TIC por parte de los dos protagonistas: estudiantes y docentes.

Tercer momento de la investigación. Diseño del modelo pedagógico fundamentado en la modalidad de educación mixta

En este tercer momento de la investigación se diseñó el modelo pedagógico fundamentado en la modalidad de educación mixta que se con base en los elementos teóricos referenciales y los elementos empíricos que surgieron producto del análisis e interpretación de las entrevistas realizadas a los informantes claves (ver Gráfico 1). 


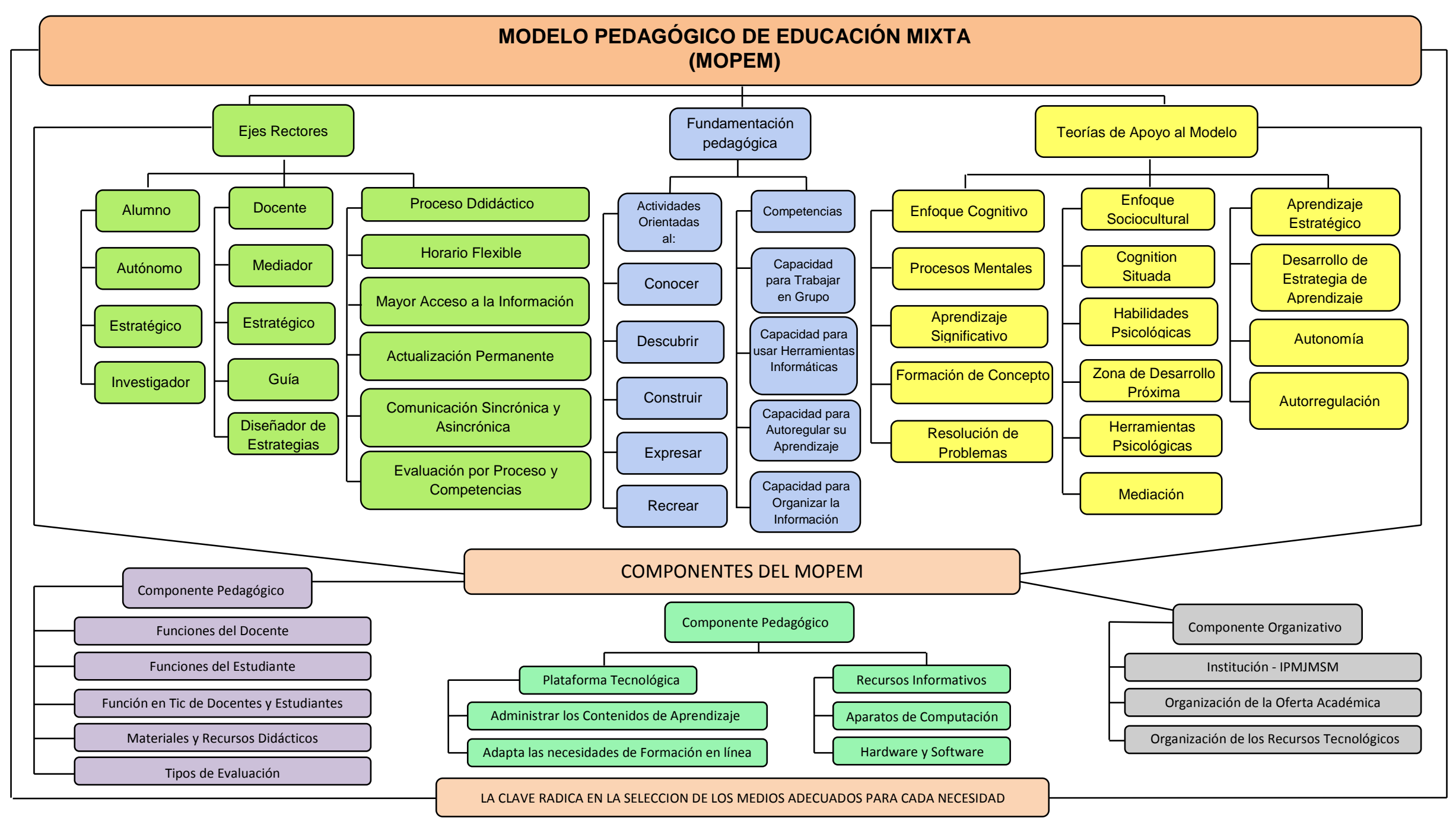

Gráfico 1. MOPEM. (Fuente: Acevedo (2019). Modelo pedagógico fundamentado en la modalidad de educación mixta para el nivel de postgrado) 


\section{CONCLUSIONES}

Sin lugar a dudas, las TIC están provocando un fuerte impacto en la educación presencial, a través de adecuados soportes tecnológicos y están permitiendo flexibilizar la educación por medio de una variedad de nuevos medios que han ido constituyendo un entorno virtual que está cambiando significativamente el proceso de enseñanza y aprendizaje. Esta nueva situación, no es solo una modificación de medios, sino que además y fundamentalmente implica una modificación sustancial en los modelos pedagógicos, en los roles de los profesores y alumnos, en el uso y comprensión del tiempo, el espacio y sobre todo en la forma de enseñar y de aprender.

De lo anterior, se desprende la importancia de un modelo pedagógico fundamentado en la modalidad mixta, la cual debe responder a los intereses y demandas de este momento histórico en los que se vive un cambio de paradigma debido al impacto que han generado el uso de las tecnologías en el ámbito de la educación. Por ello el presente estudio tuvo como propósito destacar los beneficios de combinar, integrar y complementar las sesiones presenciales con el apoyo de las TIC, según sean los escenarios y recursos; sistematizando aspectos que resumen una representación propia del Instituto Pedagógico de Miranda José Manuel Siso Martínez (IPMJMSM) para seguir recreando la modalidad, en el imperativo de seguir mejorando hacia espacios más abiertos y flexibles en el nivel de postgrado.

Con base en los planteamientos antes señalados la autora se formuló como propósito de su tesis doctoral la propuesta de un modelo pedagógico fundamentado en la modalidad de educación mixta para el nivel de postgrado (MOPEM) que promueva el uso de estrategias y recursos que ofrece la modalidad mixta como una alternativa innovadora en el proceso de enseñanza y aprendizaje de los estudios de postgrado del IPMJMSM.

Considerando los objetivos específicos planteados, se presentan a continuación las conclusiones generales del estudio.

\section{REFERENCIAS}

Acevedo, A. (2019). Modelo pedagógico fundamentado en la modalidad de educación mixta para el nivel de postgrado. Recuperado de http://espaciodigital.upel.edu.ve/jsmu i/handle/123456789/862

Cook, T. y Reichardt, Ch. (1997). Métodos cualitativos y cuantitativos en investigación evaluativa. Madrid: Morata

Del Mastro, C. (2005). Enseñanza estratégica en un contexto virtual: Un estudio sobre la formación de tutores en educación a distancia (tesis doctoral). Universidad Autónoma de Barcelona, Barcelona, España

Feo, R. (2013). Modelo Teórico para la Formación Docente centrado en el Aprendizaje Estratégico (tesis doctoral). Instituto Pedagógico de Caracas, Universidad Pedagógica Experimental Libertador, Venezuela

Gisbert, M. (2000). El profesor del siglo XXI: de transmisor de contenidos a guía del ciberespacio. En Cabero et al. (Coords.). $Y$ continuamos avanzando, las nuevas tecnologías para la mejora educativa. Kronos: Sevilla

Gros, B. y Silva, J. (2005). La formación del profesorado como docente en los espacios virtuales de aprendizaje. Revista Iberoamericana de Educación. Recuperado de 
http://www.rieoei.org/deloslectores/9 59Gros.PDF

Lobo, A. (2009). La Gestión Educativa a Distancia desde la perspectiva de la Virtualidad. Revista Una Investigación, 2(1), 76-94

Monereo, C. (2003). Estrategias para autorregular el esfuerzo en el aprendizaje. Contra el "culturismo" del esfuerzo. Revista Aula de Innovación Educativa. $\mathrm{N}^{\circ} 120,44-47$

Oficina de Planificación del Sector Universitario. (2008). Programa Nacional de Formación Docente en Educación a Distancia. Caracas: Autor

Salinas, J. (2004). Innovación y uso de las Tic en la enseñanza universitaria. Revista Universidad y Sociedad del Conocimiento, 1(1). Recuperado de http://rusc.uoc.edu/ojs/index.php/ru sc/article/viewFile/v1n1salinas/v1n1-salinas

Sangrá, A. (2002). Educación a distancia, educación presencial y usos de la tecnología: una triada para el progreso educativo. Revista Electrónica de Tecnología Educativa. $\mathrm{N}^{\mathrm{o}}$ 15. Recuperado de http://edutec.rediris.es/Revelec2/reve lec15/sangra.pdf
Sigalés, C. (2004). Formación Universitaria y TIC: nuevos usos y nuevos roles. Revista de Universidad y Sociedad del Conocimiento (RUSC). UOC, 1(1). Recuperado de http://www.uoc.edu/rusc/dt/esp/sig ales0704.pdf

Strauss, A y Corbin, J. (2002). Bases de la Investigación Cualitativa. Técnicas y procedimientos para desarrollar la teoría fundamentada. Medellín, Colombia: Universidad de Antioquia

UNESCO. (2010). Conferencia Mundial sobre Educación Superior 2009: La nueva dinámica de la educación superior y la investigación para el cambio social y el desarrollo. Ediciones Unesco, Francia. Recuperado de http://unesdoc.unesco.org/images/00 18/001832/183277s.pdf

Universidad Pedagógica Experimental Libertador, Vicerrectorado de Investigación y Postgrado. (2010). Manual de trabajos de grado de especialización y maestría y tesis doctorales (4a. ed.). Caracas: FEDUPEL

Universidad Pedagógica Experimental Libertador. (2011). Documento Base del Currículo de la UPEL. Caracas: Autor 